\title{
Advanced image processing in thermography
}

\author{
by WIECEK B. and GRECKI M.
}

Technical University of Lodz, Institute of Electronics, 18-22 Stefanowskiego str., 90-924 Lodz, Poland

\begin{abstract}
In the paper the thermal and visual camera systems working in parallel are presented. The proposed extension of widely used thermal video systems by visual camera is very helpful to identify the object elements and localise precisely hot spots on the surface. The visual camera is linked with the computer through an interface, which is based on the modern video processor in order to provide enough power to compute images in real-time. Additionally, the most suitable image processing methods for mixing images are described. The integrated software for both cameras is briefly discussed to present the potentialities of the whole system. Some experimental results confirm the useability of the developed system.
\end{abstract}

\section{Introduction}

Computer thermography gets an increasing interest in a variety of today's applications. This is caused by new developments in VLSI circuits and significant progress in image processing techniques. In consequence, it is possible an image processing of thermal and visual images in parallel. Combining both images allows to interpret the complex thermal maps easily and fast.

There are some techniques for mixing thermal and visual images [4], but it seems that the contour extraction is one of the most promising methods for improving thermal image interpretation. The main problem in constructing thermal-visual video system is to match the optical characteristics of both cameras. For that, the cameras with comparable lenses should be placed together on the same tripod (Fig.2). A powerful computer equipped with two interfaces working in parallel is recommended. A slight images' geometry modification may be performed by the computer in a software way. The visual camera interface is equipped with advanced video/graphical processor, what differentiates our solution from others and provides high computational power to process visual images in real-time.

The developed visual interface is suitable for many different thermal systems, especially for Hughes TVS-40TE or AGEMA 880. Actually, the system is running on either PC486 machine and/or portable notebook for acquiring images from the aeroplane. Some chosen system parameters are gathered in Table 1.

\section{Hardware image processing}

Typical infrared camera is an electromechanical device, where the scanning process is performed by mirrors and prisms rotation. The speed limit of this rotation causes that the thermal image generation rate is low. Additionally, because of the nature of thermal process, where the very high temperature gradients seldom exists, a low resolution data acquiring system is recommended.

Typically, resolution of 256 pixels/line is quite acceptable. As a result of this resolution the data transmission rate (from camera to the computer) does not exceed hundreds of Kbytes. One of the low-cost solutions of data capturing is based on low-density FIFO application. FIFO buffer is used to store digital data for one scanning line. There are known interfaces, where 
http://dx.doi.org/10.21611/qirt.1994.012

memory buffer does not even exist, and data is directly transferred to the computer memory in DMA channel [1].

Table 1. Some parameters of thermal and visual system

\begin{tabular}{|c|c|}
\hline $\begin{array}{c}\text { THERMAL SYSTEM - VS40TE/AGEMA880 } \\
\text { Interface and camera resolution } \\
\text { TVS40TE - } 256 \times 60, A G E M A 880-256 \times 400 \\
\text { A/D conversion resolution A/C - } 4 / 8 \text { bits } \\
\text { Registration rate } \\
\text { real-time mode (in RAM), } \\
\text { image/160ms, } \\
\text { TVS40TE - } 1 \text { mage/50ms, AGEMA880-1 } \\
\text { non real-time mode (on the hard disk), } \\
\text { repetition time } t_{\text {min }}=0.5 s \\
\text { System capacity } \\
\text { real-time mode - } 128 \text { images/1MB } \\
\text { (Hughes } 40 T E), 6 \text { images/1MB RAM } \\
\text { (Agema880) } \\
\text { non real-time mode- limited by hard disk } \\
\text { capacity }\end{array}$ & $\begin{array}{l}\text { VISUAL SYSTEM for TVS40TE/AGEMA880 } \\
\text { Visual image resolution } \\
512 \times 240 \text { (480-interlace) } \\
\text { ADD conversion resolution - } 8 \text { bits } \\
\text { Sampling rate } \\
15 \text { MHz (Quartz-controlled PLL) } \\
\text { Graphic processor } \\
\text { TMS34010-40MHz. } \\
\text { "Life-Video" mode } \\
\text { on the computer screen } \\
\text { Visual input } \\
\text { 1Vp-p (CVS), max } 4 \text { inputs } \\
\text { System capacity } \\
\text { limited by hard disk capacity }\end{array}$ \\
\hline $\begin{array}{l}\text { SVGA-256 graphics, pallets } \\
\text { images' mixing, zoom, corr }\end{array}$ & $\begin{array}{l}\text { g } \\
\text { ontrolled (keyboard) } \\
\text { ssing } \\
\text { median, noise cancelling, contours, data } \\
\text { rmal and visual image, emissivity factor } \\
7, \\
\text { is with rotation }\end{array}$ \\
\hline
\end{tabular}

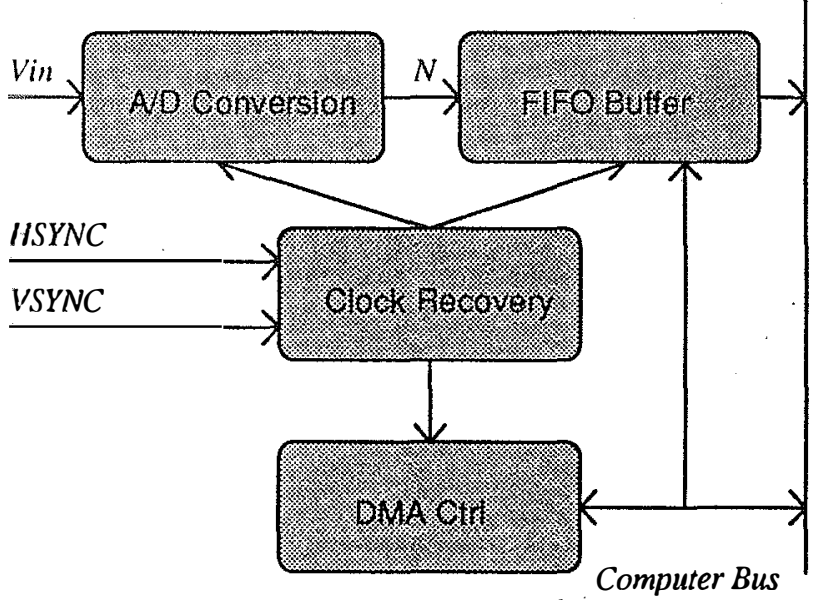

Fig.1 - Typical thermal camera interface

Sampling clock restoration circuitry is one of the main system elements. Sampling clock of high frequency is locked with horizontal and vertical deflection frequency.

Recovering of this signal can be performed with the use of digital or analogue phase locked loop. In order to get high loop stability and its noise immunity, the digital solution is always recommended. We should here emphasise that the quality of clock recovering circuitry directly corresponds to the system performance. Reassuming, thermal camera capturing systems do not require high conversion rate, but often the high accuracy of $8-12$ bits is really demanded.

Monochrome CCD camera interface (Fig.3) contains 16-bit graphic processor (TMS34010$40 \mathrm{MHz}$ ) dedicated for complex video operations. e.g.: rotation and mixing performed in reallime. The interface mentioned here has been design to co-operate with the modern dual-port memories what allows processor to access the image memory while capturing data from the 
http://dx.doi.org/10.21611/qirt.1994.012

camera. The image processor operates with its maximum speed at $40 \mathrm{MHz}$ clock rate using

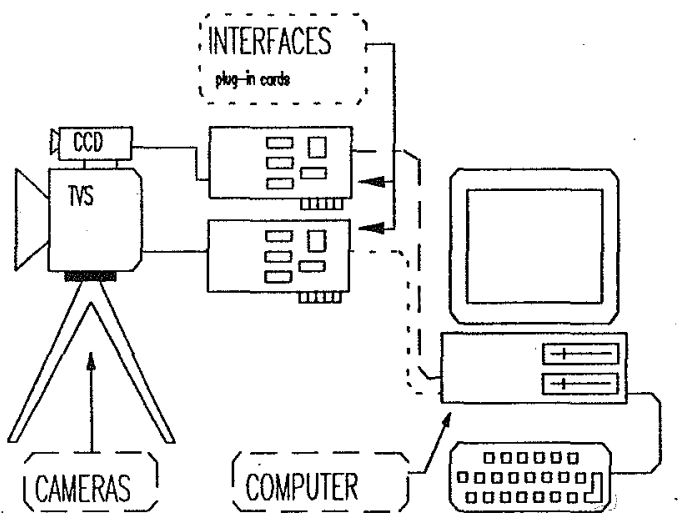

Fig. 2 - Thermal and visual system block diagram

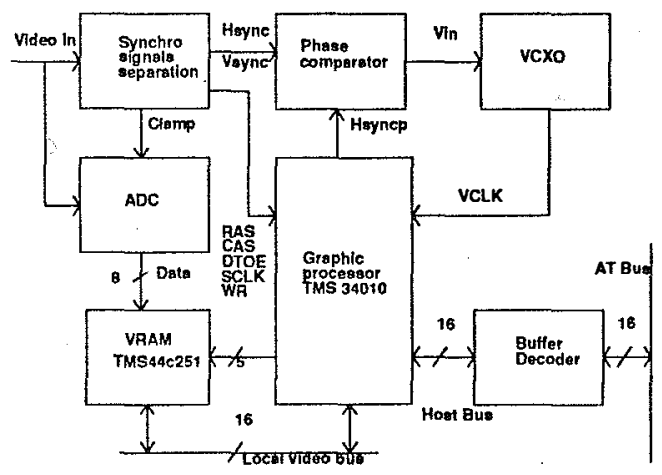

Fig. 3- $C C D$ interface block diagram pipeline and cache mechanisms.

A CRT controller included in the graphic processor is a fully programmable device, that makes possible to adjust system resolution to the application. In the realised project $512 \times 240 \quad$ (480) resolution is chosen to make compatible capturing with PC-VGA display standard. Higher vertical resolution of 480 lines per image is used while camera works in interlace mode. The entire system works under WINDOWS 3.1 control. Sampling rate of video signal at $15 \mathrm{MHz}$ ensures the square pixel and the proper image size on the screen. Video capturing circuit works with clock restoration based upon the VCXO phase locking loop (PLL). This causes the high stability of the restored sampling clock and in consequence the high quality of the captured image. VCXO-based restoration circuit is recommended in high-resolution video systems, where the slight changes in the phase clock can disturb the captured image smoothness. Additionally, the present- ed solution is using the clamping cir-cuitry for proper $A / D$ conversion being independent from the contents of the image. The entire interface has been designed with the use of programmable logic devices (PLDs) in the form of halfsize IBM-compatible computer-card. In order to fasten the transmission and capturing rate, 8/16-bit DMA transfer is applied. This feature allows to use the presented interface in so-called "Life-

video" mode, where the moving images are being displayed on the computer screen in realtime. A small size of developed interface makes possible to use it in the portable Notebook Computer. Lately, the interface has been adapted for AGEMA-880 infrared system, and now it is being used for monitoring industrial and agricültural areas from the aeroplane.

\section{Software image processing}

Main application of combined thermal and visual system is to show both images in order to make thermal image interpretation easy and fast. Mixing both images is based on contour extraction from visual image, what allows to distinguish the chosen elements in the investigated object. The method is helpful only when the luminance transitions for visual objects correspond to the temperature gradients in thermal images. This allows to localise and highlight the hot spots in thermal images. Edges can be isolated in the image by the use of an edge-enhancement/threshold algorithm. The first step - edge enhancement, extracts the luminance transitions. Next, the threshold operation generates the contour maps. One of the methods of edge enhancement is based on image filtering with the use of Sobel operator, where the enhancement image is defined by:

$$
G(i, j)=\left|F(i, j)^{*} H(i, j)\right|+|F(i, j) * V(i, j)|
$$


http://dx.doi.org/10.21611/qirt.1994.012

where: "denotes the convolution operation, $F(i, j)$ denotes the luminance of $(i, j)$-th pixel of the original image, $\mathrm{H}(\mathrm{i}, \mathrm{j})$ and $\mathrm{V}(\mathrm{i}, \mathrm{j})$ are $3 \times 3$-pixel horizontal and vertical differential operators of the following forms:

$$
H[i, j]=\left[\begin{array}{lll}
-1 / 16 & 0 & 1 / 16 \\
-1 / 4 & 0 & 1 / 4 \\
-1 / 16 & 0 & 1 / 16
\end{array}\right] \quad V[i, j]=\left[\begin{array}{ccc}
1 / 16 & 1 / 8 & 1 / 16 \\
0 & 0 & 0 \\
-1 / 16 & -1 / 8 & -1 / 16
\end{array}\right]
$$

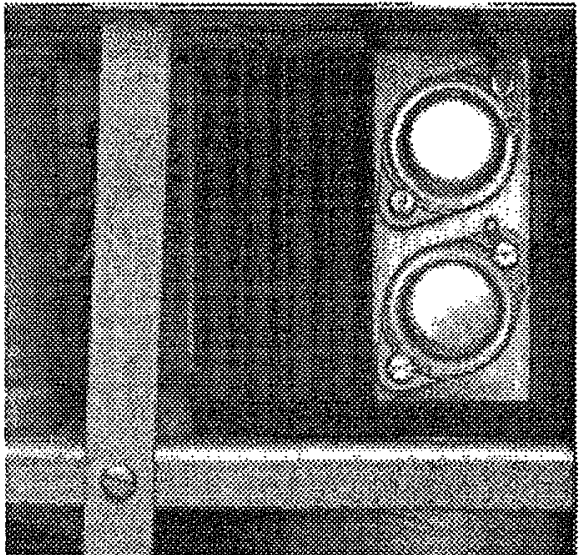

Fig. 4- Visual image of end-stage of tested power supply - power transistors

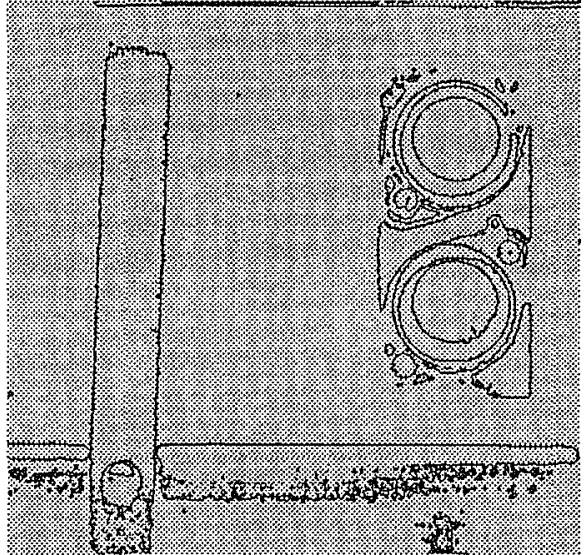

Fig. 5 - Edges' map of tested power supply

In the form of example of applying method mentioned above in electronics, we present two power transistors while working in parallel in the power supply. The original visual image and its corresponding edge map are presented in the Fig. 4 and Fig.5. In the mixed image (Fig.6), end-stage transistors which are at the highest temperature, are highlighted by surrounding them with their white edges. The superposition of images is presented in so-called pseudocolors, just for fast and easy interpretation for the user. In some applications contour extraction does not give the expected results, because of the image complexity. Too complex visual image gives very dense edges' map which can tile the thermal one and makes it unreadable [5]. In these cases just displaying two images with corrected sizes is much more worthwhile. The size adjustment is required because of the different optical systems for both cameras. The results of a such correction is presented in Fig.7.

Additionally, a correlated cursor is available, what means that by inspection of visual image we can automatically read the temperature on the thermal one.

The system has been originally prepared for HUGHES Thermal Probye Video System TVS-40TE and is equipped with two interfaces for both infra-red and video cameras. Recently, the system has been updated to co-operate with AGEMA compatible devices. The main feature of the system is to register the images in different modes. First of all, the captured images can be stored as a single object with a given repetition time varying from hundreds of milliseconds to seconds or minutes. Additionally, presented system can work in so-called realtime mode, when all images are registered in the computer memory at the rate of $50 \mathrm{~ms} / \mathrm{image}$.

High computational power allows image processing e.g.: 2-D filtering, real-time averaging, presenting thermal maps in the form of colour maps as well as 3-D diagrams. Threedimensional color diagrams give the alternate outlook of the temperature distribution. Additionally, the isotherms are available, which are very useful for the system equipped with monochrome display and printer. The emissivity correction for a given point or/and area is an useful user option [6]. In order to trace in details the temperature distribution, two linear intersections (horizontal and vertical one) are introduced. User can easily see the temperature 
http://dx.doi.org/10.21611/qirt.1994.012

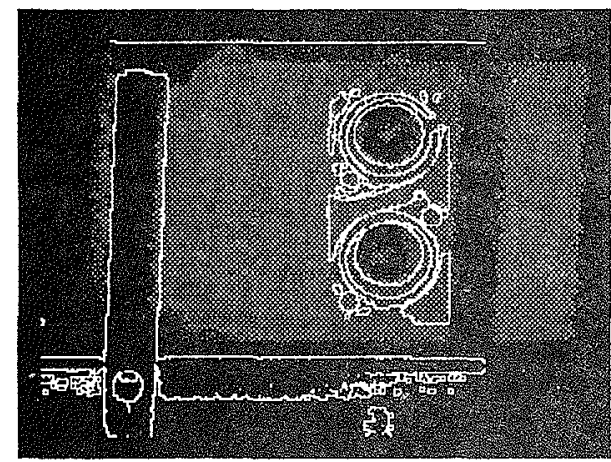

Fig.6 - Mixed image of end-stage of power supply distribution along these two perpendicular axes, which are moveable by the mouse. Twodimensional filtering has been included into the package for noise cancelling. Well known linear and non-linear filters like Sigma or Median ones have been implemented. For filtering the arithmetic co-processor is recommended to decrease the computational time.

An interesting option of histogram creating is required by some users. It allows to measure relatively, the areas of the image, which correspond to the given temperature ranges. The result is displayed in percents of total image area. The temperature can be displayed in different scales, using different

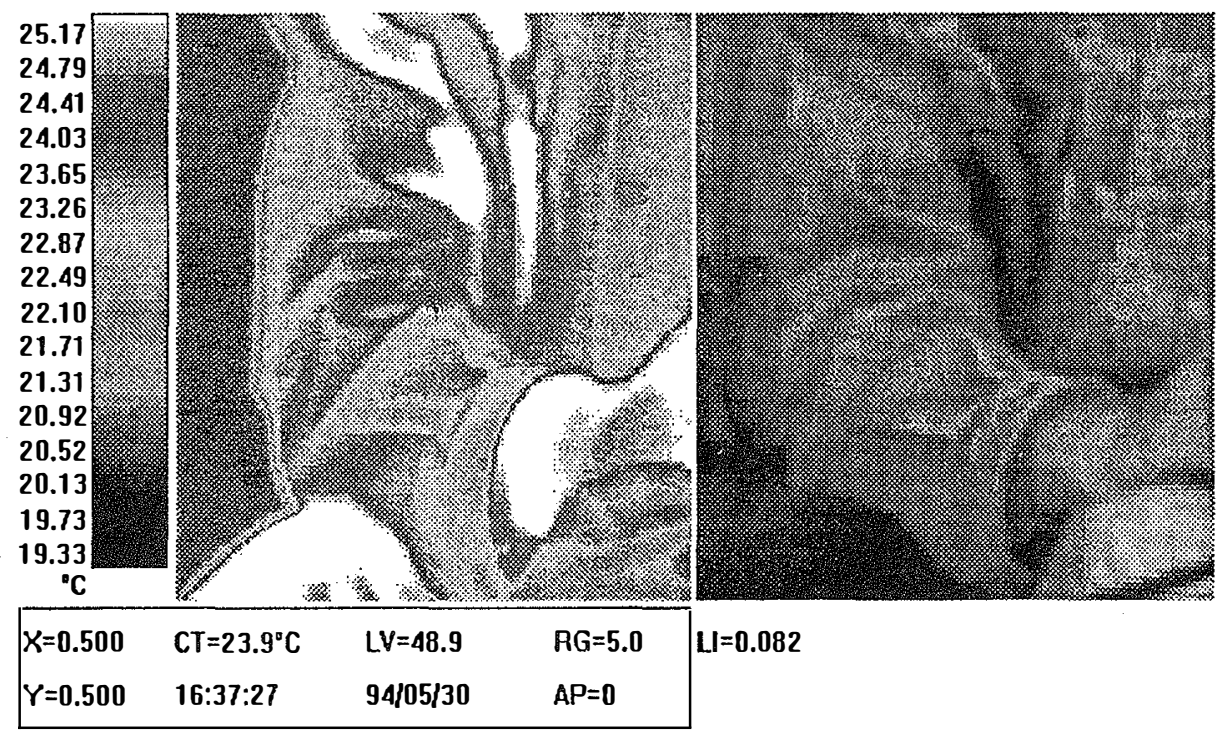

Fig. 7 - Size adjustment for visual and thermal images

user-definable pallets. In the case, when the sequence of images is being captured (repetitive or real-time acquisition), the system allows to display them in the form of the animated film on the computer screen.

\section{Conclusions}

This paper confirms the usability of combining the visual and thermal system computercontrolled together. Visual images corresponding to thermal ones allow fast and easy interpretation of thermal camera results. In the paper, the contour extraction and images' superposition are proposed as the method of image mixing. Two independent interfaces working in parallel make it possible to capture thermal and visual images at the same time. Additionally, in order to process these images in real-time, on-board fast graphic/video processor has been used. Both images operate under MS-WINDOWS control, what makes our solution very flexible for various applications. 
[1] WIECEK (B.), GRECKI (M.), PACHOLIK (J.). - Computer-based thermographic sysiem". Quantitative Infrared Thermography. Proc. Conf. QUANTITATIVE INFRARED THERMOGRAPHY, Eurotherm Seminar 27. Paris, 7-9 July 1992.

[2] WIECEK (B.), GRECKI (M.), PACHOLIK (J.). Thermal measurement of power electronic devices using thermographic system. QUANTITATIVE INFRARED THERMOGRA:HY CONFERENCE, Eurotherm Seminar 27. Paris, 7-9 July 1992.

[3] WIECEK (B.), PACHOLIK (J.), GRECKI (M.). Image Processing in Thermorgaphy". First National Conference on Thermography and its Applications. Szczyrk, Poland 1992 (in polish).

[4] PRATT (W.K.). Digital Image Processing, Willey-Interscience, New York, 1978

[5] WIECEK (B.), GRECKI (M.), PACHOLIK (J.). - Extention of Thermal Image System by Visual Camera Interface. Proc. Conf. Advanced Infrared Technology and Application, Casa Malaparte, Capri, Sept. 1993

[6] WIECEK (B.), GRECKI (M.), PACHOLIK (J.). - Emissivity Factor Evaluation Using the Reflection Method for Application in IR System in Electronics. Proc. Conf. Advanced Infrared Technology and Application, Casa Malaparte, Capri, Sept. 1993 